\title{
Exoten auf die Waage gestellt
}

\section{CERN LIBRARIES, GENEVA}

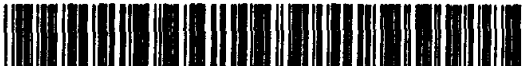

Thomas Otto

PRESSCUT-95-009

Der Massenseparator ISOLDE am CERN mit seinem breiten Spektrum von Ionenstrahlen exotischer Kerne ermöglicht sowohl Massenbestimmungen an extrem kurzlebigen Atomkernen fernab der Stabilität als auch Präzisionsexperimente an langlebigeren Kernen zur Verbesserung von Kernmodellen.
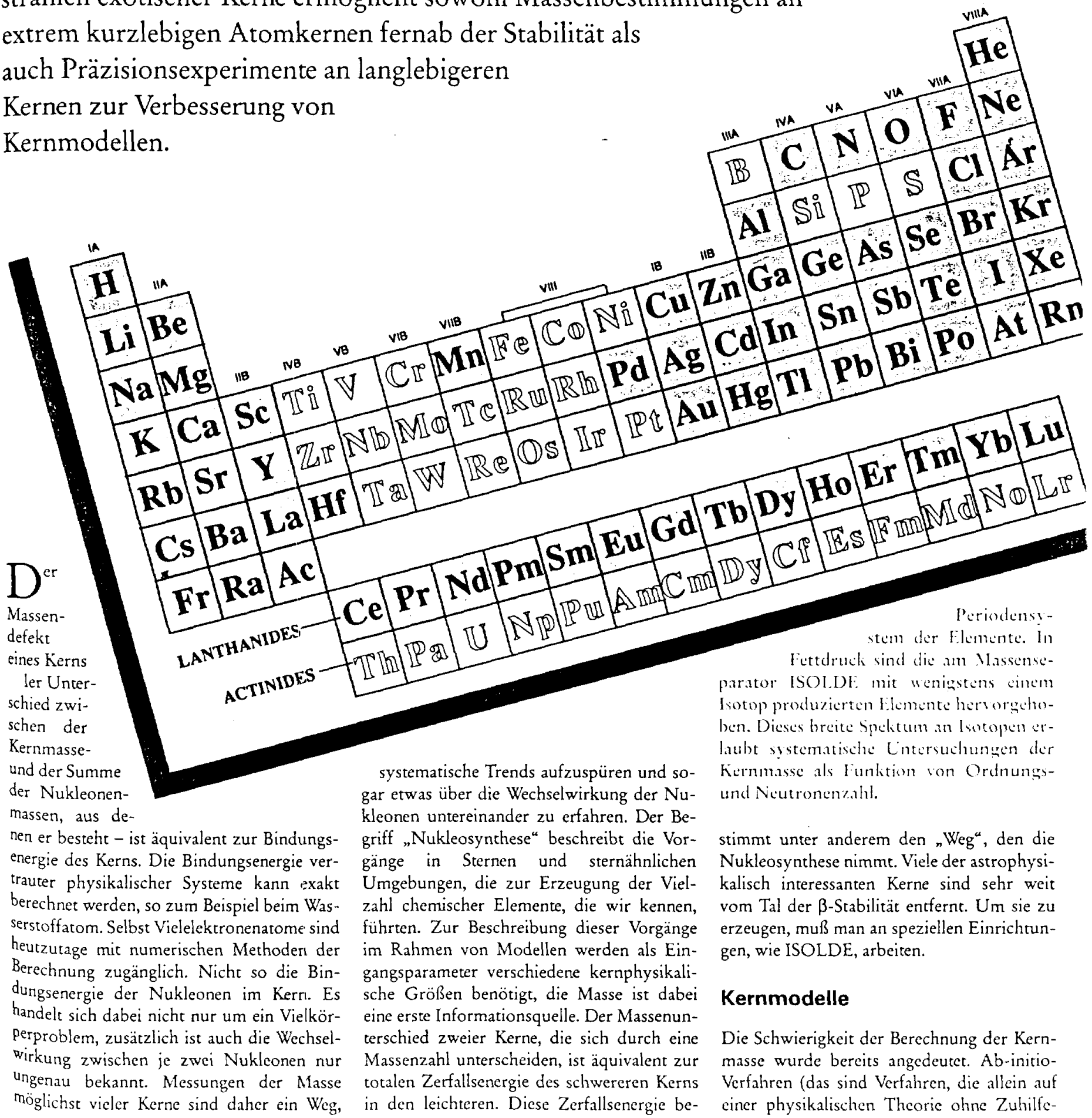

stimmt unter anderem den "Weg ${ }^{a}$, den die Nukleosynthese nimmt. Viele der astrophysikalisch interessanten Kerne sind sehr weit vom Tal der $\beta$-Stabilität entfernt. Um sie zu erzeugen, muß man an speziellen Einrichtungen, wie ISOLDE, arbeiten.

\section{Kernmodelle}

Die Schwierigkeit der Berechnung der Kernmasse wurde bereits angedeuter. Ab-initioVerfahren (das sind Verfahren, die allein auf einer physikalischen Theorie ohne Zuhilfe-

\footnotetext{
Physik in unserer Zeit / 26. Jahrg. 1995 / Nr. 1

O VCH Verlagsgesellschiff mbH, 69469 Weinhem, $1995 \quad 0031-9252 / 95 / 0101-002955.00+.2510$
} 

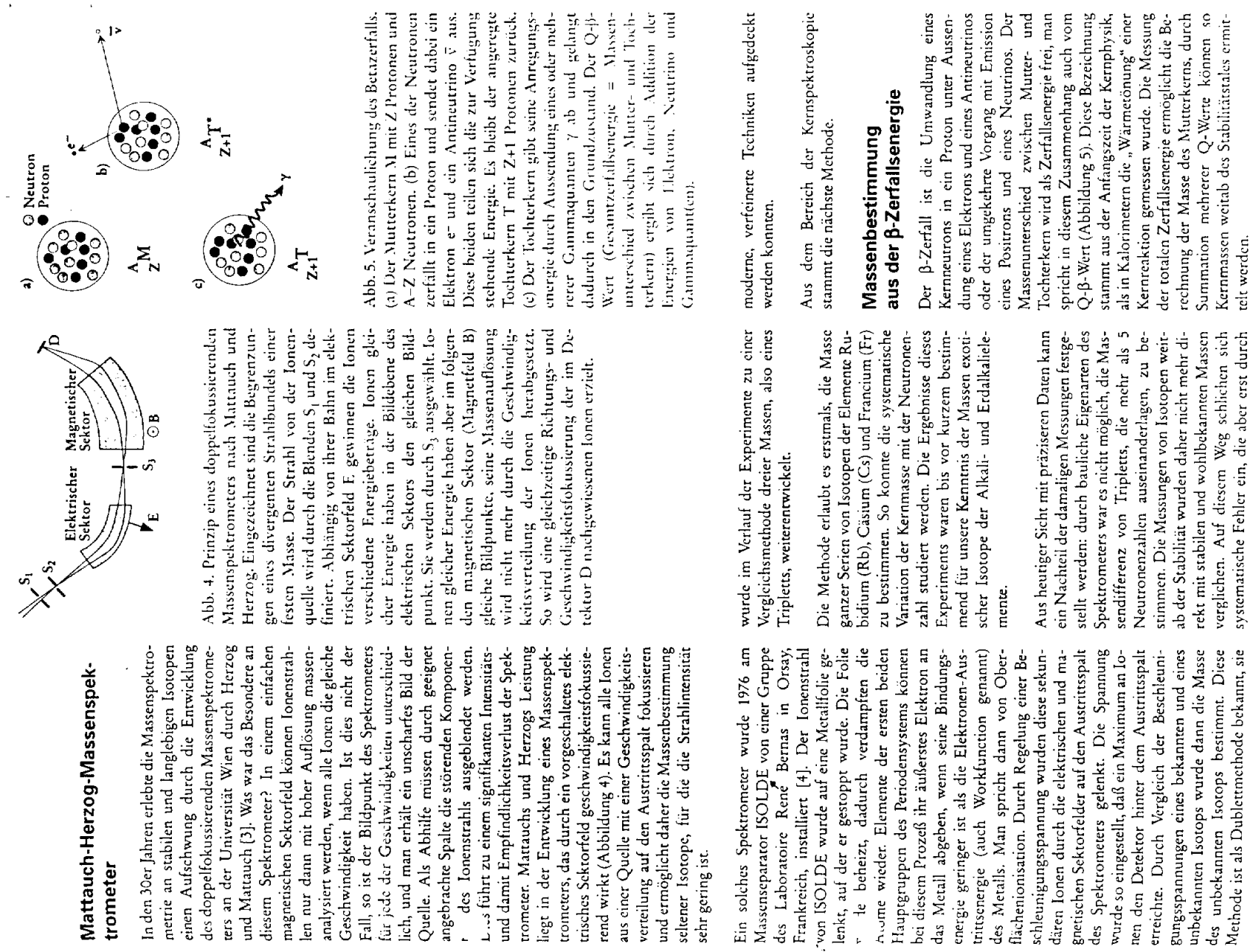

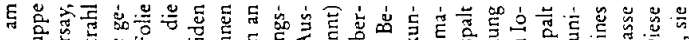

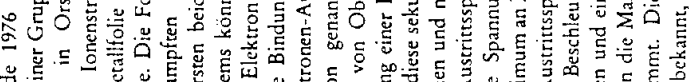

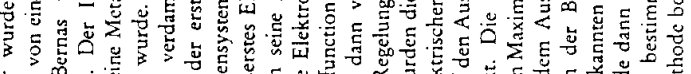

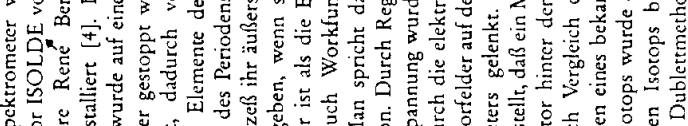

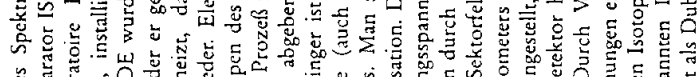

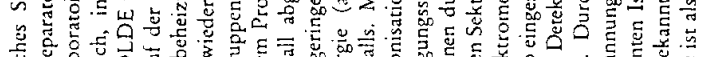

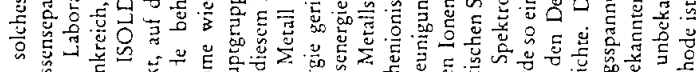

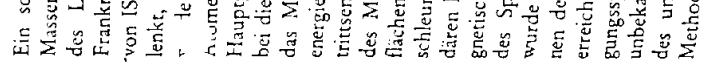

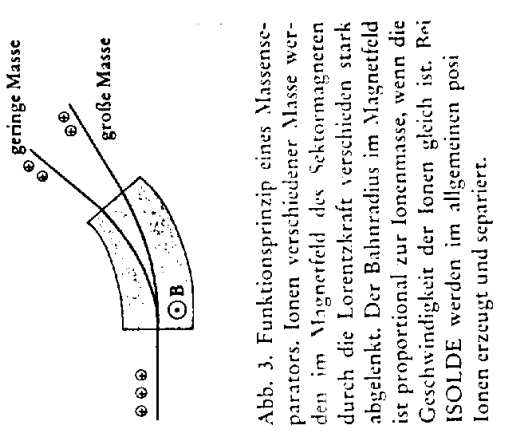

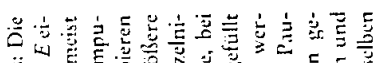

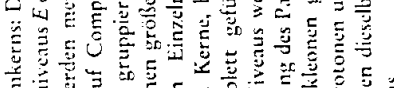

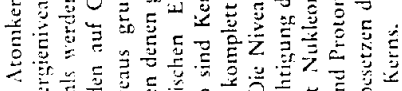

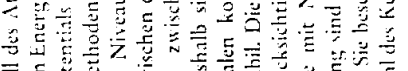

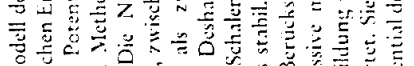

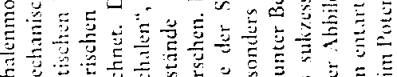

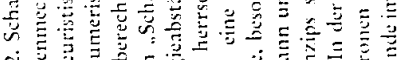

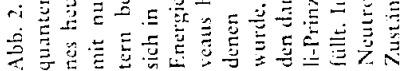

(1)

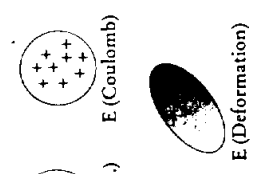

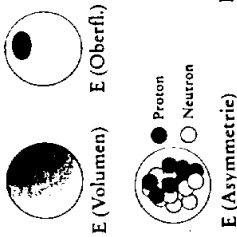

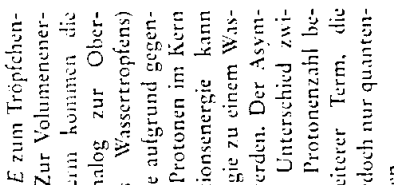

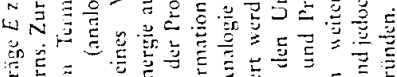

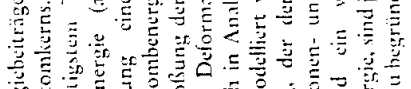

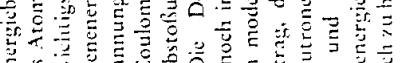

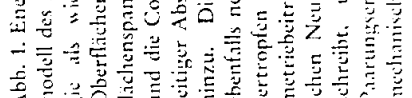

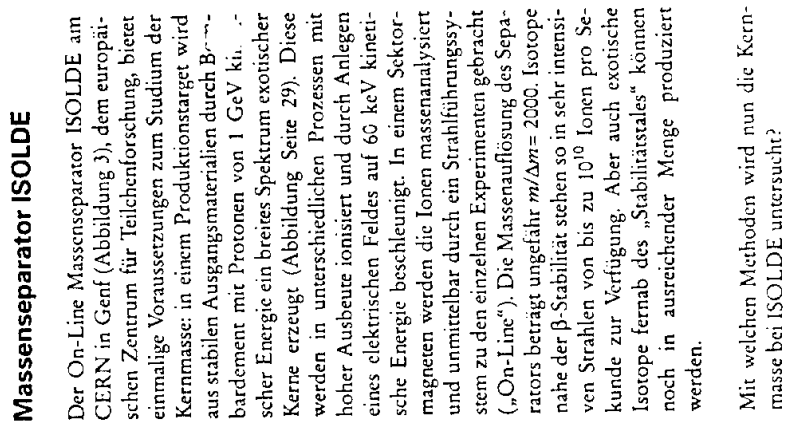

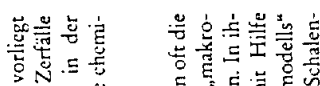

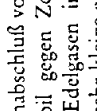

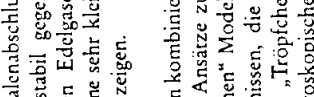

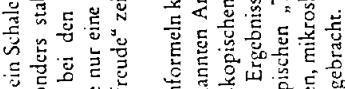

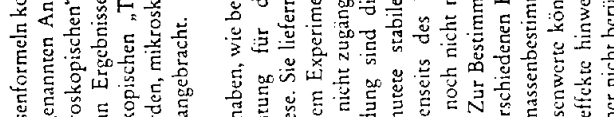

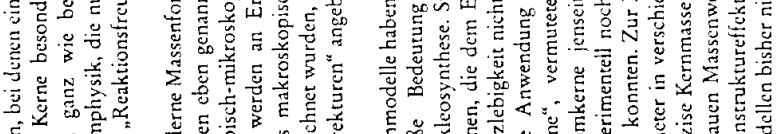

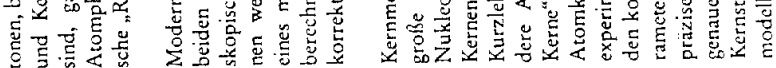

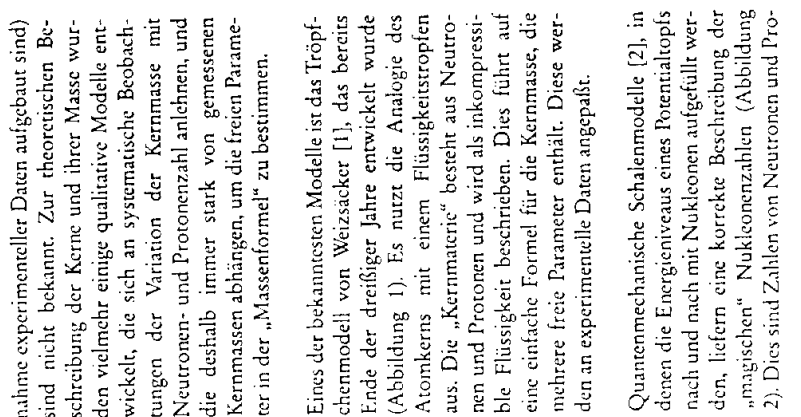



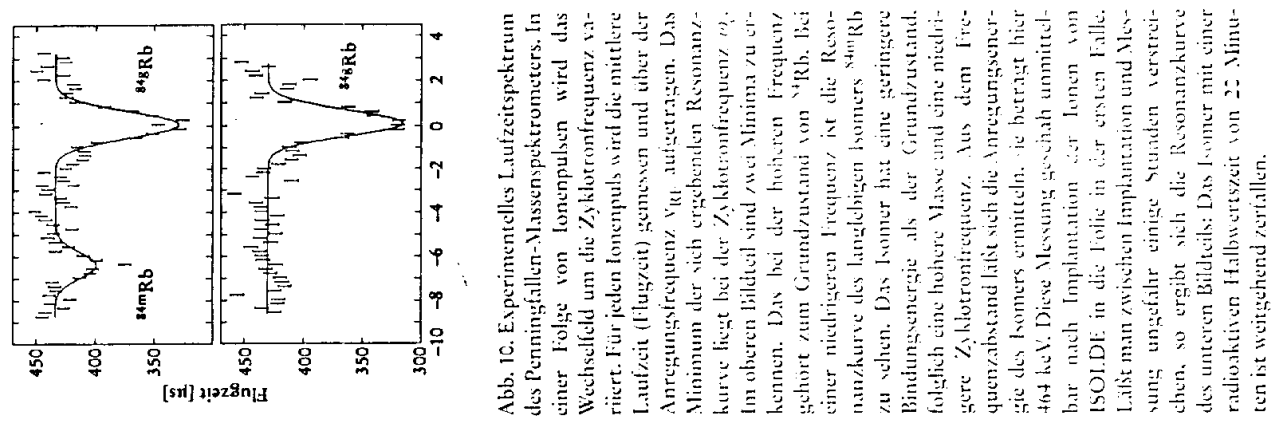

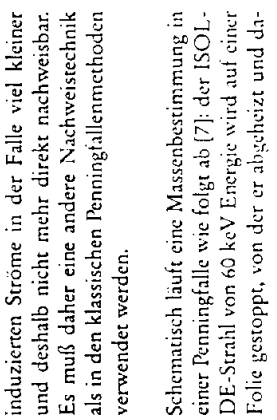

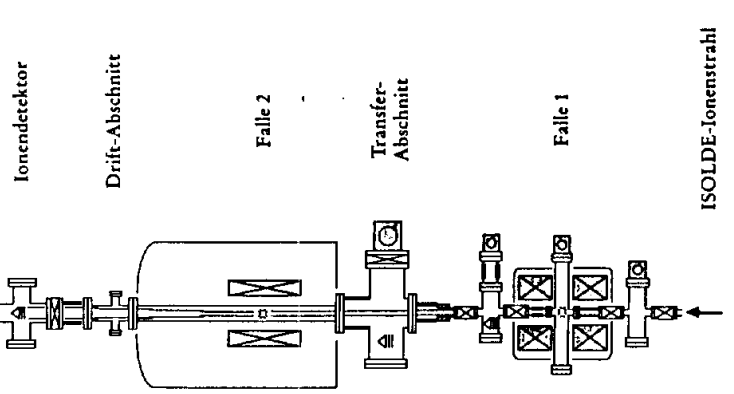

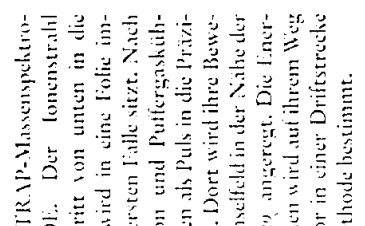

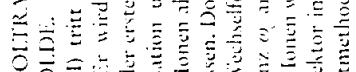

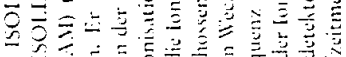

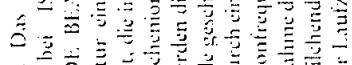

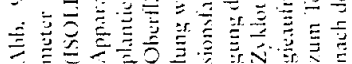

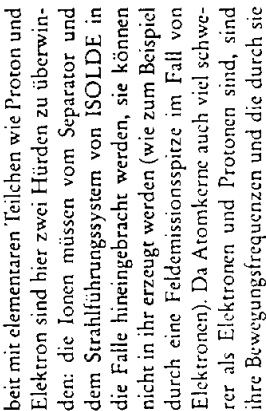

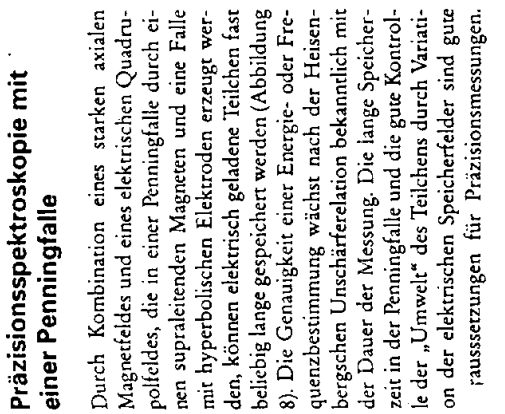

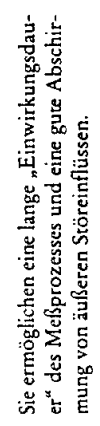

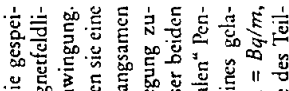

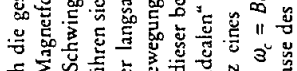

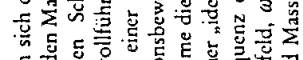

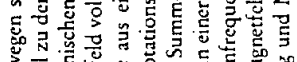

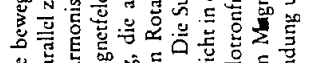

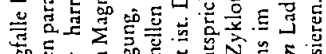

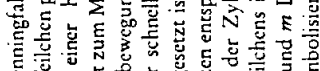

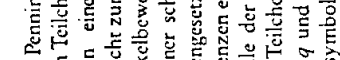

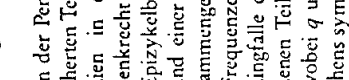

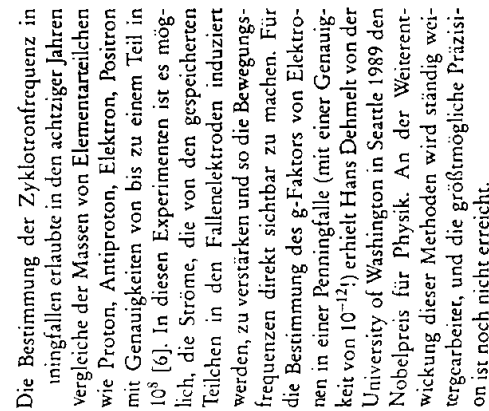

总宝宫安

总壳总

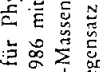

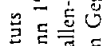

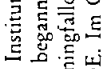

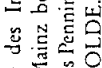

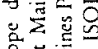

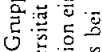

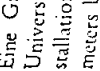
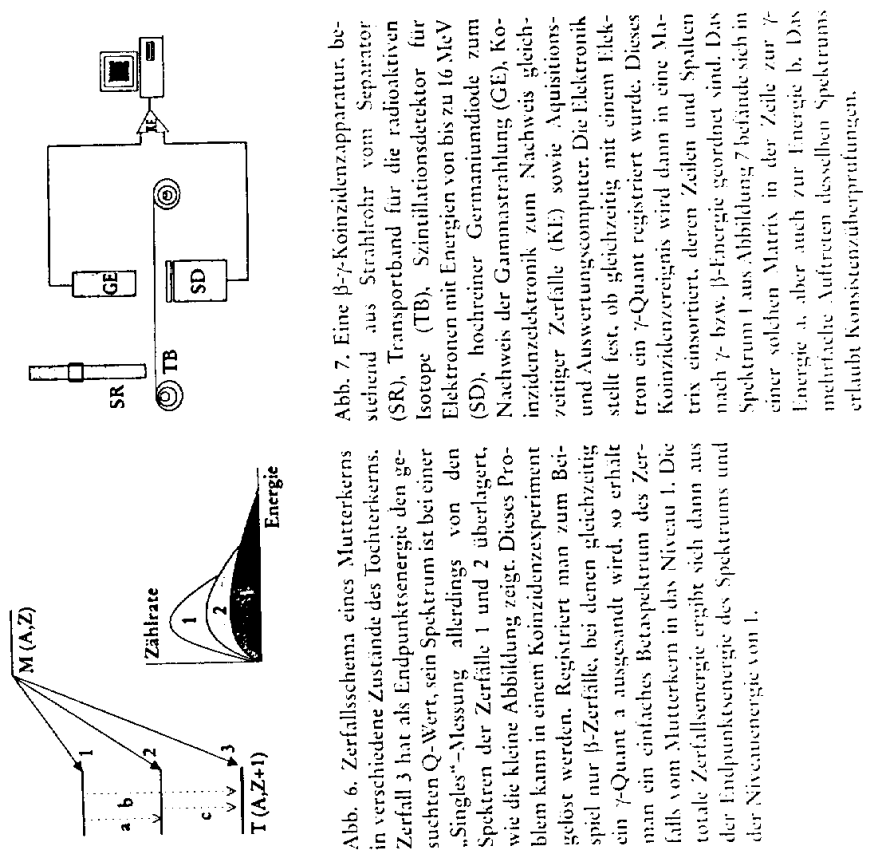

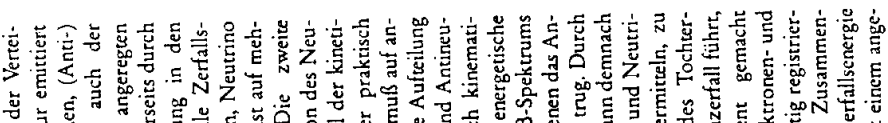

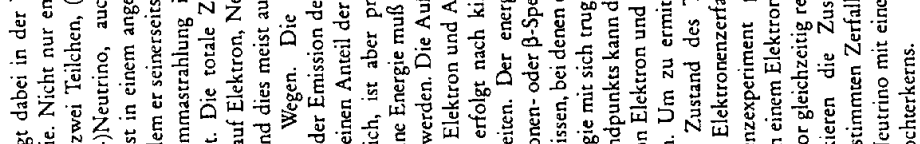

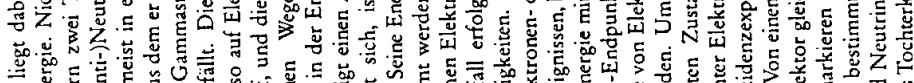

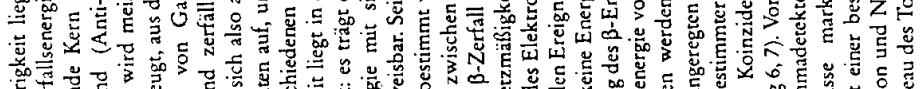

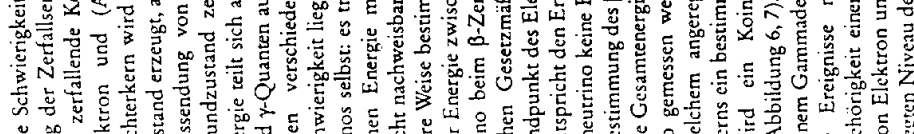

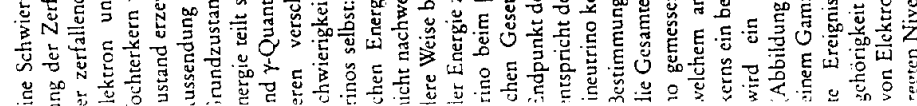
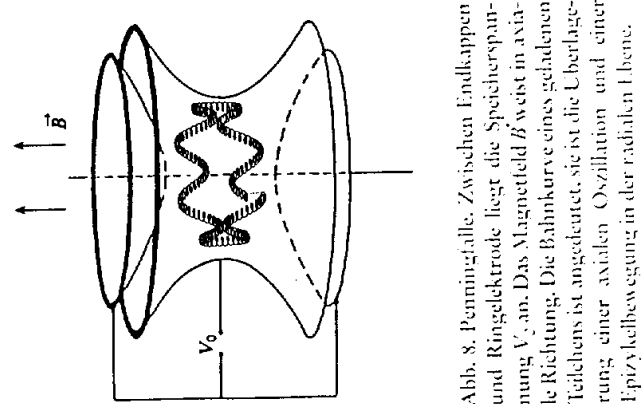

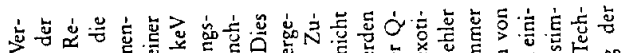

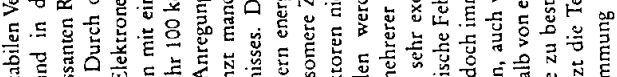

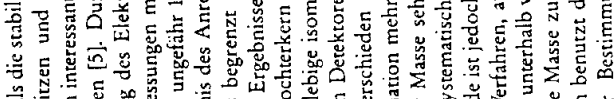

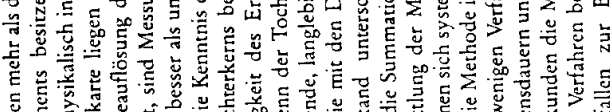

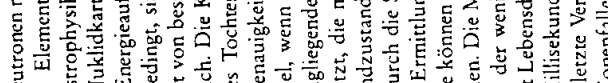

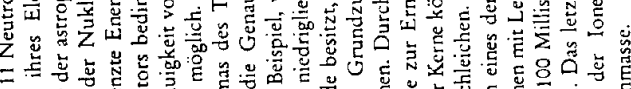

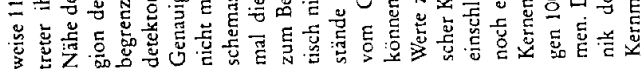

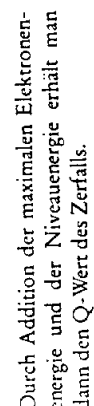

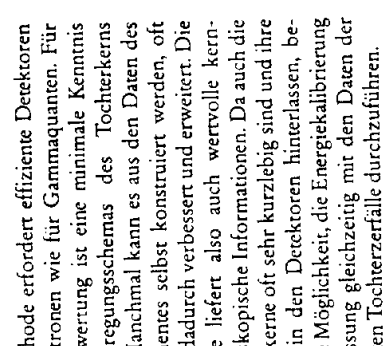

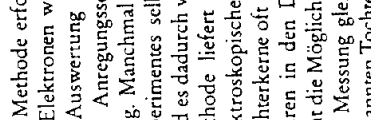

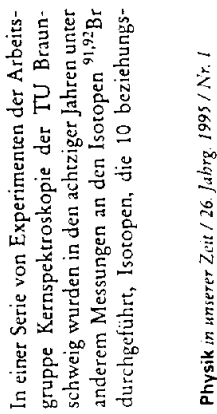


bei oberflächenionisiert wird. Dies geschieht in der ersten von zwei Penningfallen. Die Ionenwolke wird in ihr durch Stöße mit einem Puffergas gekühlt und dann als Puls ausgeschossen. Dieser Puls kann in der zweiten Falle, der Präzisionsfalle, dynamisch eingefangen werden, ungefähr wie in einer Mausefalle. Die Ionenbewegung in der Präzisionsfalle wird durch ein Wechselfeld in der Nähe der Zyklotronfrequenz $\omega_{c}$ angeregt. Die Energieaufnahme der Ionen ist dann am größten, wenn die Anregungsfrequenz mit der Zyklotronfrequenz übereinstimmt, das heißt, mit ihr in Resonanz ist (Abbildung 10). Dieser Energiezuwachs wird durch ein Flugzeitverfahren nachgewiesen: Die Ionen werden aus der Falle in Richtung auf einen Detektor ausgeschossen. Erfüllte das angelegte Wechselfeld die Resonanzbedingung, so erreichen sie den Derektor schneller, weil sie Energie aufgenommen haben. Die Kalibrierung der Daten erfolgt durch die Messung der Zyklotronfrequenz eines stabilen Isotops mit bekannter Masse auf die gleiche Weise. Es handelt sich also wieder um eine Dublettmethode. Die Massenauflösung ist umgekehrt proportional zur Wechselwirkungszeit des Anregungsfeldes mit dem gespeicherten Ion. Sie ist so hoch, daß es gelang, die Zyklotronfrequenzen des Grundzustandes und eines isomeren Zustandes in ${ }^{84} \mathrm{Rb}$ zu trennen ( $\mathrm{Ab}$ bildung 10). Die Fehler der Massenbestimmung liegen aufgrund von Kalibrationsunsicherheiten ungefähr bei $10^{-7}$, das entspricht etwa $10 \mathrm{keV}$ bei Massenzahl 100. Es gibc keine Aufsummierung von Fehlern, da alle Frequenzen direkt mit der des stabilen Kalibrierungskerns verglichen werden.

\section{In Zukunft: noch vielseitiger, noch kurzlebiger}

Wie geht es weiter mit der Massenspektrometrie an ISOLDE? Von den beschriebenen Experimenten ist nur das letzte noch aktiv. Es befindet sich im Augenblick (1994) in einer Umbauphase. Die erste der zwei Penningfallen wird gegen eine Neukonstruktion ausgetauscht, die es erlauben wird, auch Ionen, die mittels kurzer Laserpulse erzeugt wurden, einzufangen, zu kühlen und in die Präzisionsfalle weiterzutransportieren [8]. In den bisherigen Experimenten war die Technik auf oberflächenionisierbare Elemente der ersten beiden Hauptgruppen des Periodensystems beschränkt. Durch die Erweiterung mit einer Laserdesorptions-Ionenquelle wird es nicht nur möglich, eine Vielzahl von den bei ISOLDE verfügbaren Isotopen der Messung zugänglich zu machen. Durch die Erzeugung und Massenbestimmung von Kohlenstoffclustern als Referenz werden dann auch absolute Massenbestimmungen möglich, da Kohlenstoff die Basis des Kernmassensystems darstellt.

Die einzige Einschränkung bildet die Lebensdauer der Atomkerne: je kürzer diese ist, desto geringer kann aufgrund der Unschärferelation nur die maximal erreichbare Frequenzauflösung und damit die Meßgenauigkeit sein. Schließlich kann die Wechselwirkung zwischen Anregungsfeld und gespeichertem Ion nicht länger als dessen Lebensdauer sein. Außerdem dauert auch die Präparation der Ionen für die eigentliche Messung in der Falle eine gewisse Zeitspanne in der Größenordnung von einer Sekunde, so daß diese Zahl, außer für Spezialfälle, die untere Grenze der Lebensdauer für "Routinekerne“ darstellen dürfte.

Eine französische Kollaboration schlug kürzlich den Einstz eines Smith-Massenspektrometers [9] vor. In diesem Spektrometer, das ursprünglich für die Untersuchung der Antiprotonenmasse konstruiert wurde, wird mit einem zur Penningfalle alternativen Verfahren die Zyklotronfrequenz von lonen im magnetischen Feld bestimmt. Im Smith-Spektrometer geschieht das bei einer einzigen Passage der Ionen durch ein homogenes Magnetfeld, die nur Mikrosekunden dauert. Um trotzdem eine hohe Auflösung zu erzielen, funktioniert der Nachweis bei einer sehr hohen Harmonischen von $\omega_{c}$. Die Präzision des Spektrometers wird für leichte Elemente vorraussichtlich bei der der Penningfalle liegen. Gerade in dieser Massenregion liegen die Halbwertszeiten exotischer Kerne deutlich unter einer Sekunde, sie sind daher nicht gut geeignet für die Untersuchung in einer Penningfalle. Die Installation des Smith-Spektrometers würde deshalb eine wertvolle Ergänzung des massenspektrometrischen Forschungsprogramms an ISOLDE darstellen.

\section{Literatur}

[1] C.F. v. Weizsäcker, Z. Phys. 96, 431 (1935)

[2] M. Mayer, Phys. Rev. 75, 1969 (1949)

[3] J. Mattauch, R. Herzog, Z. Phys. 89, $736(1934)$

[4] M. Epherre, G. Audi, C. Thibault, R. Klapisch, G. Huber, F. Touchard und $H$.
Wollnik, Phys. Rev. C19, 1504 (1979)

[5] M. Graefenstedt, U. Keyser, F. Münnich und F. Schreiber, Nucl. Phys. A491, 373 (1989)

[6] G. Bollen und C. Carlberg (Ed.), 'Workshop on Physics with Penning Traps', Physica Scripta 46, 255 (1992), Physica Scripta 46, 544 (1992), Physica Scripta 47, 470 (1993)

[7] H. Stolzenberg, St. Becker, G. Bollen, F. Kern, H.-J. Kluge, Th. Otto, G. Savard, G. Audi, B. Moore and the ISOLDE Collaboration, Phys. Rev. Lett. 65, 3104 (1990)

T. Otto, G. Bollen, G. Savard, L. Schweik hard, H. Stolzenberg, G. Audi, R.B. Moore, G. Rouleau, J. Szerypo, Z. Patyk and the ISOLDE Collaboration, Nucl. Phys. A567, 281 (1994)

[8] G. Bollen, H. Hartmann, H.-J. Kluge, M. König, Th. Otto, G. Savard, H. Stolzenberg and the ISOLDE Collaboration, Physica Scripta 46, 581 (1992)

\section{[9] L.G. Smith, Phys. Rev. C4, 22 (1971)}

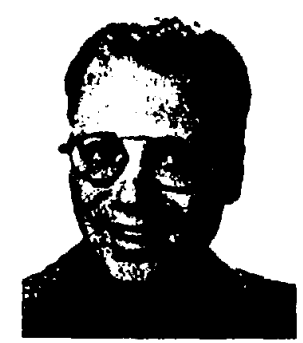

Der Autor:

Thomas Otto, Jahrgang 1962, arbeitete nach seinem Diplom in Kernspektroskopie am ISOLTRAP-Massenspektrometer am CERN. Promotion 1993 an der Universität Mainz, Postdoc an der Pennsylvania State University, zur Zeit wissenschaftlicher Assistent am Institut de physique nucléaire.

\section{Anschrift:}

Dr. Thomas Otto, Institut de physique nucléaire, Chemin de Cyclotron 2, B-1348 Louvain-La-Neuve, Belgien. 